\title{
Host conditioning and rejection monitoring in hepatocyte transplantation in humans
}

Kyle A. Soltys ${ }^{\star 1}$, Kentaro Setoyama*2, Edgar N. Tafaleng ${ }^{\star 2}$, Alejandro Soto Gutiérrez ${ }^{3,4}$, Jason Fong $^{2}$, Ken Fukumitsu ${ }^{3}$, Taichiro Nishikawa ${ }^{2}$, Masaki Nagaya ${ }^{2}$, Rachel Sada ${ }^{1}$, Kimberly

Haberman ${ }^{1}$, Roberto Gramignoli ${ }^{5}$, Kenneth Dorko ${ }^{3}$, Veysel Tahan ${ }^{3}$, Alexandra Dreyzin ${ }^{2}$, Kevin Baskin $^{6}$, John J. Crowley ${ }^{6}$, Mubina A. Quader ${ }^{7}$, Melvin Deutsch ${ }^{7}$, Chethan Ashokkumar ${ }^{1}$, Benjamin L. Shneider ${ }^{8}$, Robert H. Squires ${ }^{8}$, Sarangarajan Ranganathan ${ }^{9}$, Miguel Reyes-

Mugica $^{9}$, Steven F. Dobrowolski ${ }^{9}$, George Mazariegos ${ }^{1}$, Rajavel Elango ${ }^{10}$, Donna B. Stolz $z^{4,11}$, Stephen C. Strom ${ }^{5}$, Gerard Vockley ${ }^{12}$, Jayanta Roy-Chowdhury ${ }^{13,14}$, Marilia Cascalho ${ }^{15}$, Chandan Guha ${ }^{16}$, Rakesh Sindhi ${ }^{1}$, Jeffrey L. Platt ${ }^{15}$, Ira J. Fox ${ }^{1,2,4}$

1. Thomas E. Starzl Transplant Institute, Children's Hospital of Pittsburgh of UPMC, Pittsburgh, PA

2. Department of Surgery, University of Pittsburgh School of Medicine, Pittsburgh, PA

3. Department of Pathology, University of Pittsburgh School of Medicine, Pittsburgh, PA

4. McGowan Institute for Regenerative Medicine, University of Pittsburgh School of Medicine, Pittsburgh, PA

5. Department of Laboratory Medicine, Division of Pathology, Karolinska Institutet, Stockholm, Sweden

6. Division of Vascular and Interventional Radiology, Children's Hospital of Pittsburgh of UPMC, Pittsburgh, PA

7. Department of Radiation Oncology, University of Pittsburgh School of Medicine, Pittsburgh, PA

8. Division of Pediatric Gastroenterology, Hepatology, and Nutrition, Children's Hospital of Pittsburgh of UPMC, Pittsburgh, PA

9. Department of Pathology, Children's Hospital of Pittsburgh of UPMC, Pittsburgh, PA

10. Department of Pediatrics, University of British Columbia and Child \& Family Research Institute, BC Children's Hospital, Vancouver, Canada

11. Department of Cell Biology and Physiology, University of Pittsburgh School of Medicine, Pittsburgh, PA

12. Departments of Pediatrics and Human Genetics, University of Pittsburgh School of Medicine and Department of Medical Genetics, Children's Hospital of Pittsburgh of UPMC

13. Departments of Medicine and Genetics, Albert Einstein College of Medicine, Bronx, NY

14. Marion Bessin Liver Research Center, Albert Einstein College of Medicine, Bronx, NY

15. Departments of Surgery and Microbiology and Immunology, University of Michigan, Ann Arbor, MI

16. Department of Radiation Oncology, Albert Einstein College of Medicine, Bronx, NY

*These authors share co-first authorship

Corresponding Author: Ira J. Fox, M.D.; Children's Hospital of Pittsburgh of UPMC, 4401 Penn Avenue, Pittsburgh, PA 15224; phone: 412-692-6693; fax: 412-692-6599; e-mail:

ira.fox@chp.edu

Electronic Word Count:

Abstract: 249 words

Manuscript (Abstract + Text + Figure Legends + References): $\underline{\text { 9,335 words }}$

Number of Figures: 8

Number of Supplementary Figures: 5

Number of Tables: 0

Number of Supplementary Tables: 1 
List of Abbreviations: CD154, cluster of differentiation 154; PKU, phenylketonuria; PHE, phenylalanine; IR, immune reactivity index; NHP, non-human primate; CT, computed tomography; IMRT, Intensity Modulated Radiation Therapy; IRB, Institutional Review Board; FDA, Food and Drug Administration; UCD, urea cycle disorder; MV, megavoltage; PBL, peripheral blood lymphocytes; anti-CD8, antibody against cluster of differentiation 8; antiCD154, antibody against cluster of differentiation 154; HLA, human leukocyte antigen; antiCD25, antibody against cluster of differentiation 25; DNA, deoxyribonucleic acid; PCR, polymerase chain reaction; CPS1, carbamoyl-phosphate synthase-1; OTC, ornithine transcarbamylase; LCU, luminescent counting unit; FISH, fluorescence in situ hybridization; DSA, donor-specific antibody; PAH, phenylalanine hydroxylase; IQ, intelligence quotient; FRG, Fah-/-/Rag2-/-/II2rg-/-; uPA SCID, urokinase plasminogen activator severe combined immunodeficiency; ADHD, attention deficit hyperactivity disorder

Keywords: Hepatocyte transplantation; Liver-based metabolic disease; Liver-directed radiation therapy; Phenylketonuria; Rejection risk monitoring; Hepatocyte xenotransplantation

Conflict of Interest: The authors declare no funding from industries or conflict of interest with respect to this manuscript.

Financial Support: This work was supported by grants DoD W81XWH-11-1-0803, NIH R01 Al49472 and NIH R01 Al122369 (IJF), NIH HL52297 and NIH R01 Al122369 (JLP), NIH R01 DK064670 and NIH R33 CA121051 (CG), NIH R01 DK092469 (JR-C) and NIH R01 DK099257 (AS-G). This work was also supported by funds from the Children's Hospital of Pittsburgh Foundation, the Children's Hospital of Pittsburgh of UPMC, and the Department of Surgery and McGowan Institute for Regenerative Medicine, University of Pittsburgh School of Medicine.

Author's Contributions:

KAS, KS, ENT, CG, JR-C, AS-G, GV and IJF designed the clinical trial, NHP transplants, and analysis. RS, RHS, GV and IJF wrote the IRB proposal. RSa was the clinical research coordinator for regulatory affairs and FDA compliance. RSa, KH, KAS, AS-G, JJC, BLS, GM and IJF were involved in the medical management of patients. JF, KF, TN, MN, and KS were involved in daily management and surgery of NHPs and pigs. MAQ, MD and CG performed CT planning and delivery of radiation therapy. RG, KD, VT, and SCS isolated donor hepatocytes and participated in the transplant procedures. KAS, RS, AS-G, KB, JJC, GM and IJF were key participants to the transplant procedures. RHS and CA performed the immunoreactivity studies, SR and MR-M performed histology and immunocytochemistry, SFD performed DNA analysis, and RE performed the whole body PHE turnover analysis of clinical samples. JLP and MC performed immunologic studies, DS performed immunocytochemistry, and ENT performed DNA analysis of NHP samples. KAS, KS, ENT, AD, RS, AS-G, GV, JLP and IJF wrote the manuscript. All authors reviewed, critiqued and offered comments to the text. 
Background: Hepatocyte transplantation partially corrects genetic disorders and has been associated anecdotally with reversal of acute liver failure. Monitoring for graft function and rejection has been difficult, and has contributed to limited graft survival.

Methods: Preparative hepatic irradiation was examined in non-human primates as a strategy to improve engraftment of donor hepatocytes, and was then applied in human subjects. T-cell immune monitoring was also examined in human subjects to assess adequacy of immunosuppression.

Results: Porcine hepatocyte transplants engrafted and expanded to comprise up to $15 \%$ of irradiated segments in immunosuppressed monkeys preconditioned with 10Gy liver-directed irradiation. Two patients with urea cycle deficiencies had early graft loss following hepatocyte transplantation; retrospective immune monitoring suggested the need for additional immunosuppression. Preparative radiation, anti-lymphocyte induction, and frequent immune monitoring were instituted for hepatocyte transplantation in a 27-year old female with classical phenylketonuria. Post-transplant liver biopsies demonstrated multiple small clusters of transplanted cells, multiple mitoses, and Ki67+ hepatocytes. Mean peripheral blood phenylalanine (PHE) level fell from pre-transplant levels of $1343 \pm 48 \mu \mathrm{M}$ (normal $30-119 \mu \mathrm{M}$ ) to $854 \pm 25 \mu \mathrm{M}$ (treatment goal $\leq 360 \mu \mathrm{M})$ after transplant $(36 \%$ decrease; $p<0.0001)$, despite transplantation of only half the target number of donor hepatocytes. PHE levels remained below $900 \mu \mathrm{M}$ during supervised follow-up, but graft loss occurred after follow-up became inconsistent.

Conclusions: Radiation preconditioning and serial rejection-risk assessment may produce better engraftment and long-term survival of transplanted hepatocytes. Hepatocyte xenografts engraft for a period of months in non-human primates and may provide effective therapy for patients with acute liver failure.

\section{Lay summary}

Hepatocyte transplantation can potentially be used to treat genetic liver disorders but its application in clinical practice has been impeded by inefficient hepatocyte engraftment and the inability to monitor rejection of transplanted liver cells. In this study, we first show in non-human primates that pretreatment of the host liver with radiation improves the engraftment of transplanted liver cells. We then used this knowledge in a series of clinical hepatocyte transplants in patients with genetic liver disorders to show that radiation pretreatment and rejection risk monitoring are safe and, if optimized, could improve engraftment and long-term survival of transplanted hepatocytes in patients. 


\section{Introduction}

Transplantation of isolated hepatocytes has been proposed as a minimally invasive alternative to organ transplantation for acute liver failure and liver-based metabolic deficiencies [1]. Hepatocytes have been safely transplanted into the liver, have produced partial correction of Crigler-Najjar syndrome type 1 [2], factor VII deficiency [3], urea cycle disorders (UCD) [4, 5], infantile Refsum's disease [6], glycogen storage disease type 1 [7], and phenylketonuria (PKU) [8], and have been associated anecdotally with reversal of acute hepatic failure [1]. Because of an inability to transplant an adequate cell mass, monitor graft function, or diagnose rejection, disease correction has been incomplete, the function of transplanted cells has been short-lived, and the need for organ transplantation has not been averted.

Preparative liver-directed irradiation was developed as a strategy for enhancing the initial engraftment and expansion of donor hepatocytes in architecturally normal host livers after transplantation [9]. Studies in rodents demonstrate that radiation conditioning targeting only a portion of the liver [10] and in modest doses [11], leads to complete correction of the bilirubin conjugation defect in the Gunn rat model of Crigler-Najjar syndrome type I following hepatocyte transplant [10].

The random nature of engraftment also produces great variability in the distribution of donor cells, creating difficulty in diagnosing donor hepatocyte rejection on liver biopsy. In addition, measurement of hepatic functional changes mediated by the transplanted cells, such as bilirubin, amino acid or ammonia levels, has not been sensitive enough to detect rejection of donor cells before damage to the allograft is irreversible and the allograft is lost. This inability to diagnose and treat rejection may result in over- or under-immunosuppression and silent graft loss using standard immunosuppression regimens. We have previously evaluated allospecific 
CD154+ T-cytotoxic memory cells (CD154+ TcM) and their subsets to assess rejection risk for acute cellular rejection in children with liver or intestine transplants, and in adult renal transplant recipients. In those studies [12-14], test results were reported as a numerical score, the immune reactivity index (IR), that predicts acute rejection within a 60-day time period after sampling with sensitivities ranging from $84-88 \%$ and specificities ranging from $80-88 \%$ in both children and adults.

Here we report that porcine hepatocytes delivered into the portal circulation of immunosuppressed monkeys preconditioned with 10Gy liver-directed irradiation targeting the right lobe, enter the liver in large numbers, engraft and expand to comprise up to $15 \%$ of irradiated segments. This preparative radiation regimen and use of donor-specific CD154+ Tcell immune monitoring was then used following hepatocyte transplantation in an infant with CPS1, in an infant with OTC and in a 27-year old female with classical PKU in an attempt to enhance donor hepatocyte engraftment and more closely monitor graft survival.

\section{Materials and methods}

\section{Animal Studies}

\section{Animals}

Male cynomolgus monkeys (Macaca fascicularis) weighing between $6.7 \mathrm{~kg}$ and $9.0 \mathrm{~kg}$ were used as transplant recipients. The animals were kept at the Rangos Research Center, Children's Hospital of Pittsburgh of UPMC, University of Pittsburgh School of Medicine. Longdwelling single lumen venous catheters were inserted into the internal jugular vein and central lines were tunneled subcutaneously to exit through the skin in the interscapular region of the animals' backs. Jackets were used to protect the indwelling central IV line. Hepatocytes were isolated from Yorkshire piglets, 6-8 weeks of age (8 kg to $15 \mathrm{~kg}$ ) (Wally Whippo, Enon Valley, 
PA). In one experiment, Landrace piglets genetically engineered as homozygous $\alpha 1,3-$ galactosyltransferase knockout (Revivicor, Inc., Blacksburg, VA) were used as the source of hepatocytes. All studies were approved by the University of Pittsburgh Institutional Animal Care and Use Committee and thus within the guidelines for humane care of laboratory animals.

\section{Radiation treatment of non-human primates (NHPs)}

Animals were anesthetized with isoflurane and placed on a CT table and immobilized with an individually shaped Vac-Lok vacuum pillow (Civco, Orange City, lowa). Irradiation (10Gy) was delivered using $6 \mathrm{MV}$ photon energy beams after 3-dimensional computerized tomographic treatment planning via a combination of coplanar and non-coplanar portals that were shaped by multi-leaf collimation to limit the dose to normal structures like kidney, spinal cord, and small bowel. Dose-volume histograms were generated from the computerized treatment planning (Supplementary Fig. 1A, B).

\section{Intrahepatic liver cell transplantation in NHPs}

A midline incision was made $1-2 \mathrm{~cm}$ below the xiphoid process. The small bowel mesentery was brought into the field and a 20-gauge angiocath was inserted into a mesenteric vessel, and a 0.018 guide wire was advanced through the angiocath into the main portal vein. The angiocath was then replaced with a $4 \mathrm{f}$ intravascular sheath and a portal venogram was obtained via the sheath to determine the vascular anatomy. A 3 f Fogarty thrombectomy catheter (Edwards Lifesciences, Irvine, CA) was introduced over the guide wire and advanced into the main right portal vein. Fogarty catheters were chosen as inflation of the balloon resulted in proximal occlusion. Cells could then be directed into the desired hepatic lobe without refluxing into nontarget areas of the liver. Occlusion of the portal venous flow was performed to produce relative ischemia to the target lobe, which would aid in donor cell proliferation. Maintenance of portal 
flow integrity was confirmed every 15 minutes during the infusion by performing contrast portography to identify shunting or flow stasis of the portal circulation (Supplementary Fig. 2B). Fresh porcine hepatocytes in $60 \mathrm{ml}$ of Dulbecco's modified Eagle medium were injected through the portal vein catheter. The catheter was then removed and mesenteric vessel bleeding was controlled with ligatures of 3-0 silk.

\section{Clinical Studies}

Patient enrollment

The ClinicalTrials.gov identifier for the transplants are NCT01345578 and NCT01465100. Approval to perform hepatocyte transplantation in patients with liver-based metabolic deficiencies was obtained from the University of Pittsburgh School of Medicine Institutional Review Board and from the Food and Drug Administration (FDA) under Investigational New Drug license 6880. The hepatocyte-transplantation protocol was explained to patients and their families, and written informed consent for participation was given.

\section{Host preconditioning and transplantation of hepatocytes}

Once hepatocytes with acceptable viability were isolated, patients underwent conformal radiotherapy to the right lobe of the liver using Intensity Modulated Radiation Therapy (IMRT). CT based treatment planning for radiation therapy was performed after informed consent for hepatocyte transplantation was signed. The target volume was $30-37 \%$ of the total liver volume. The IRB and FDA approved protocol prescribed a total dose of 10Gy for patients greater than 3 years of age, and 5Gy for patients less than that age. As the first recipient was 4 months at the time of transplant, the dose administered was $5 \mathrm{~Gy}$ in a single fraction to the first child treated with a UCD. As this patient had no untoward effects from radiation, permission was given to increase the dose to $7.5 \mathrm{~Gy}$ for the second patient of 7 months treated with a UCD. The adult 
patient with PKU received 10Gy in a single fraction. The target volume and location were determined so as to keep the dose administered to extra hepatic tissues as low as possible. Both children were irradiated under general anesthesia, whereas the adult patient did not require anesthesia. Radiation was delivered with 6 megavoltage (MV) photons using 9 and 7 coplanar beams, respectively (Fig. 1). Out of three patients, two were treated with motion-gated radiotherapy to minimize dose critical structures; for the third patient, gating during treatment was not necessary. The accuracy of target placement was confirmed using an on board conebeam CT imager.

Fresh allogeneic hepatocytes were infused into the liver through the portal vein. Access to the left portal vein was established in all three patients by making an incision just above the umbilicus and recanalizing the umbilical vein remnant using coronary dilators as previously described [15]. A 5 French arterial sheath was placed into the umbilical vein and patients were systemically heparinized. The right portal vein was selected using a 5 French Kumpke angled catheter (Cook Medical, Bloomington, IN). A 3 or 4.5 French Fogarty thrombectomy catheter (Edwards Lifesciences, Irvine, CA) was introduced into the right portal vein and, following contrast injection to confirm satisfactory catheter positioning, portal venous pressures were measured. Fogarty catheters were chosen, as inflation of the balloon resulted in proximal occlusion. Cells could then be directed into the desired hepatic lobe without danger of cells refluxing into non-target areas of the liver. Occlusion of the portal venous flow was performed to produce relative ischemia to the target lobe, which would aid in donor cell proliferation [16]. Maintenance of portal flow integrity was confirmed every 15 minutes during the infusion by measuring portal vein pressures and by performing contrast portography to identify flow stasis or porto-systemic shunting (Fig. 2). The patient's hemodynamic and cardiopulmonary status was continuously monitored. A total of $2.0 \times 10^{8}$ viable cells $/ \mathrm{kg}$ were prepared for each patient. 
Transplantation was performed over approximately 26 hours in three to four infusions separated by 6-8 hours. Hepatocyte aliquots were infused over 40 minutes on each occasion during portal occlusion.

\section{Cellular immune monitoring}

CD154+ TcM were used to measure donor-specific alloreactivity in a 16-hour mixed lymphocyte culture at $37^{\circ} \mathrm{C}$ in $5 \% \mathrm{CO}_{2}$, as described previously [14]. In this assay, recipient peripheral blood lymphocytes (PBL) pre-labeled with fluorochrome-labeled anti-CD8 antibody are co-cultured 1:1 with donor or third-party PBL in culture medium containing anti-CD154-phycoerythrin. This approach facilitates non-permeabilizing intracellular detection of expressed CD154. If donor lymphocytes were not available at the time of analysis, PBL from normal human subjects that match the donor human leukocyte antigen (HLA) at a minimum of one antigen each at the HLAA, -B and -DR loci were used. Third-party lymphocytes are HLA-mismatched to donor and recipient at all loci. Results are expressed as IR or the ratio of donor-induced to third-partyinduced CD154+ TcM. Based on previous work, an IR $\geq 1.13$ was used as a target value implying increased risk of rejection $[12,13]$. Test results were matched to clinical data retrospectively to determine whether changes in graft function coincided with or were preceded by changes in the risk of acute cellular rejection.

\section{Results}

Optimizing liver-directed preparative radiation therapy in NHPS

Having previously established the liver's sensitivity to irradiation in cynomolgus monkeys through dose escalation studies, we selected a radiation dose of 10Gy, well below the threshold for liver or systemic toxicity [17]. Three-dimensional CT-based treatment planning was performed for delivery of intensity modulated radiation therapy to the right lobe of the liver. 
Using this approach significant delivery of radiation to the remaining liver or surrounding structures was minimized. The best combination of coplanar and non-coplanar treatment portals was determined, and dose-volume histograms were generated. The beams were shaped by multi-leaf collimation or by custom-made blocks so that the transmission of radiation was less than $5 \%$ below the blocks to limit the dose to normal structures (Supplementary Fig. 1A, B).

The 10Gy of irradiation to the right lobe was administered in a single fraction and comprised 36$50 \%$ of the total liver volume (Supplementary Fig. 1B, C). Ten percent of the right kidney received less than $5 \mathrm{~Gy}$ and $50 \%$ received $\leq 0.2 \mathrm{~Gy}$. The adjacent spinal cord and small bowel received less than 3.9Gy of irradiation (Supplementary Fig. 1B). Long term experience in treating infants with symptomatic liver hemangiomas and Wilms' tumor indicated that the risk of acute and late sequelae from this dose of liver irradiation was exceptionally low [18-21].

\section{Porcine hepatocyte transplantation in NHPs}

One day after liver-directed irradiation, porcine hepatocytes were isolated as described in Supplementary Methods and in our prior reports [22]. In two experiments the hepatocytes were isolated from wild type pigs, and in one experiment the hepatocytes were isolated from a pig with a disrupted $\alpha 1,3$-galactosyltransferase locus [23], and hence deficient in the Gal 1 1-3Gal saccharide recognized by human natural antibodies [24]. For each transplant, $6.0-7.5 \times 10^{8}$ viable porcine hepatocytes, suspended in Plasmalyte, were infused into the portal vein of NHPs

(Supplementary Fig. 2A). During the hepatocyte infusion, the NHPs, anesthetized with isoflurane, were treated with heparin, and a balloon was used to transiently occlude the portal circulation, as we previously found that the ischemia so induced evokes compensatory regenerative signals that together with irradiation facilitate engraftment of hepatocytes. Infusion of hepatocytes was discontinued when shunting of blood flow from the portal system to the 
hepatic veins and inferior vena cava was demonstrated during intermittent portal venography during the procedure. Severe pruning of the portal veins was seen prior to portal to hepatic vein shunting (Supplementary Fig. 2B). The NHPs were treated with an immunosuppression regimen consisting of Thymoglobulin $\AA$, methylprednisolone, and anti-CD25 at the time of transplantation (induction) and with cyclosporine, FTY720, everolimus and methylprednisolone thereafter, as detailed in Supplementary Methods.

Two days after transplantation, porcine albumin was detectable in the blood of the preconditioned recipients but not in an unconditioned recipient (Supplementary Fig. 2C). Since normal levels of endogenous (monkey) albumin in the blood of recipients may impair the sensitivity of the immunoassay for the porcine protein, potentially confounding its use as an objective measure of engraftment, we used immunohistochemistry and molecular analysis of tissue samples to evaluate the level of engraftment. Without radiation preconditioning, hepatocyte transplantation after complete embolization of the left portal branch and right anterior portal branch leads to only about $1-2 \%$ engraftment $(1.8 \% \pm 0.2 \%$ at 16 weeks) [16]. Because only transient occlusion of the portal vein was performed in our studies, we expected the level of engraftment to be about $1 \%$ with transient balloon ischemia alone. Spatial analysis of stained sections of tissues obtained at the termination of the experiments (28-45 days after transplantation) showed that porcine hepatocytes comprised $11.8 \pm 2.4 \%$ of the irradiated right lobes and $5.0 \pm 0.1 \%$ of the region of the unirradiated left lobes adjacent to the irradiated right lobes (Fig. 3A-D and Supplementary Fig. 3). The level of engraftment in the unirradiated liver lobes notably exceeds the level usually observed in unconditioned recipients (1-2\%) [16] and we suspect it reflects overflow of radiation to the median lobe. Consistent with engraftment of porcine hepatocytes in the irradiated lobe, porcine-specific albumin DNA sequences were detected by genomic PCR (Supplementary Fig. 4). Since replacement of $\sim 5 \%$ of liver mass by 
transplanted hepatocytes appears to allow effective treatment of metabolic diseases in humans and acute liver failure in rodents [2, 25, 26], the level of engraftment achieved with preconditioning should suffice to confer benefit in a clinical setting.

The survival of hepatocyte xenografts in preconditioned NHPs given a "clinical" immunosuppression regimen is consistent with our previous finding that hepatocyte xenografts escape the rejection reactions that occur during transplantation of whole-organ xenografts [22]. Indeed, histologic analysis of liver specimens from preconditioned recipients revealed no evidence of rejection. Quantitative immunopathology revealed few $(<0.1 \%)$ infiltrating T cells or macrophages (Supplementary Fig. 5A-D). The recipients did have anti-Gala1-3Gal antibodies in the circulation at the time of transplantation (Supplementary Fig. 5E); however, the levels of these antibodies decreased in recipients of hepatocytes producing that sugar, suggesting that the antibodies were adsorbed to the grafts or bound to secreted glycoconjugates [27]. Importantly, none of the recipients had detectable antibodies against antigens other than Gal $\alpha 1$ 3Gal, suggesting immunosuppression constrained T-cell-dependent B-cell responses. The absence of evidence of rejection, and the progressive expansion of porcine hepatocytes suggests the regimen of immunosuppression controlled the immune response of the recipient to an adequate extent.

\section{Treatment of infants with CPS1 and OTC deficiency}

Based on the above experience in NHPs, two children with urea cycle defects received radiation conditioning prior to hepatocyte transplantation and were also monitored for rejection risk using an assay that measures allospecific CD154+ TcM. Donor livers in both of these recipients were 
from identical blood group donors. B and T cell cross-matches were negative and Luminex testing did not reveal circulating donor-specific HLA antibodies.

Hepatocyte transplantation was performed at 4 months of age in the first child, who was a female diagnosed with carbamoyl-phosphate synthetase 1 (CPS1) deficiency shortly after birth. Despite optimal management, the patient suffered several hyperammonemic crises in the neonatal period resulting in multifocal changes on brain MRI. She was managed on standard ammonia conjugation therapy, including oral sodium phenylbutyrate or IV sodium phenylacetate/benzoate (Ammonol) and arginine supplementation.

The patient received $5 \mathrm{~Gy}$ of radiation therapy to the right lobe of the liver as pre-transplant host conditioning once the quality and quantity of the donor cells was deemed adequate for transplantation. The total area irradiated constituted $33 \%$ of the liver mass. The first infusion of hepatocytes was performed four to six hours after radiation therapy was given, and two subsequent hepatocyte infusions were given over the following 26 hours. The patient did not exhibit any adverse events from the hepatocyte transplant procedure or the radiation therapy treatment. A total of $6.89 \times 10^{8}$ viable hepatocytes were infused $\left(1.25 \times 10^{8}\right.$ viable cells $/ \mathrm{kg}$ body weight). Post-isolation hepatocyte viability was $88 \%$ (Fig. 4A), and progressively decreased to $63 \%$ during the 26 hours required to perform the three infusions. Apoptosis level, measured in term of Caspase $3 / 7$ activities, was 43.4 and $38.8 \mathrm{LCU} / \mathrm{min} / 10^{3}$ viable cells at the first and last injection, respectively (Fig. 4B), values considered within the normal range based on historical controls [28]. Specific hepatic functions measured at first and last infusion showed phase I and ammonia metabolism within or superior to the normal range (Fig. 4C). Immunosuppression consisted of methylprednisolone, Tacrolimus (targeted trough level 10-12 ng/mL), and mycophenolate mofetil (15mg/kg twice a day). 
Postoperatively, the ammonia and glutamine levels increased slightly, most likely affected by high dose glucocorticoid administration during the peri-transplant period. Once these levels returned to baseline, dietary protein was slowly advanced. Despite receiving donor hepatocytes the patient continued to have intermittent episodes of hyperammonemia (Fig. 5A).

Unfortunately, goal Tacrolimus levels were not met as the mean Tacrolimus level for the posttransplant course was $8.9 \pm 4.7 \mathrm{ng} / \mathrm{mL}$. Immunologic studies were performed at three different time points. On the day of hepatocyte infusion, the IR was elevated at 1.35 , and a repeat IR, on post-transplant day 14 , was increased to 1.86 . The IR was repeated on post-transplant day 30 , when it was measured at 1.15. Donor-specific antibodies were not detected. Since the patient continued to have episodes of high ammonia despite being on limited protein intake and full dietary medical support, immunosuppression was withdrawn and the patient received a liver transplant three and one-half months after hepatocyte transplant. Histologically there was no evidence of radiation changes to the right lobe of the explanted native liver. Because the patient had been off immunosuppression for some time, we thought that the transplanted cells were no longer present by this time and therefore did not attempt to detect transplanted cells in the explant.

The second recipient underwent hepatocyte transplantation at 7 months of age. He was diagnosed with ornithine transcarbamylase (OTC) deficiency as a neonate following a hyperammonemic crisis. The child was maintained on protein restriction, Buphenyl or Ammonol, and citrulline, but experienced numerous hyperammonemic episodes, occasionally requiring dialysis for resolution. He also had numerous central IV access line infections and had significant developmental delay. 
The patient received $7.5 \mathrm{~Gy}$ of radiation therapy to the right lobe of the liver, still constituting $37 \%$ of the liver mass as preconditioning. The first infusion of hepatocytes was performed four hours after motion gated radiation therapy was given. A total of $2.0 \times 10^{9}$ viable hepatocytes were transplanted $\left(2.0 \times 10^{8}\right.$ viable cells $/ \mathrm{kg}$ body weight) over four infusions. Post-isolation hepatocyte viability was $76.9 \%$ (Fig. 4A), and the whole procedure required 16 hours with pauses between injections equal to 8,2 and 4 hours, respectively, resulting in cell viability of $56 \%$ at the last injection. Apoptosis levels at the beginning and end of the procedure were 23.2 and 17.5 $\mathrm{LCU} / \mathrm{min} / 10^{3}$ viable cells, in line with our historical control group (Fig. 4B). Hepatic metabolism was within the normal range during the infusions, and after long-term culture (Fig. 4C). Based on the assumption that graft loss was the result of rejection from inadequate immunosuppression at the time of transplant in the first patient, perioperative basiliximab was added to the immunosuppression regimen. No adverse events occurred during the transplant procedure or in the immediate post-operative course.

Glutamine and ammonia levels increased slightly in the perioperative period, as seen in the first patient, but then returned to baseline. Beginning on day 20 after transplant dietary protein was advanced. Beginning around 40 days after transplant, however, intermittent increases in the ammonia level required return to severe dietary protein restriction, and an acute increase in ammonia, glutamine and urinary orotic acid levels on post-transplant day 84 suggested loss of the hepatocyte graft (Fig. 5B). IR measurements were performed at several time points. The IR was 0.84 on post-infusion day 4 , and 0.62 on post-infusion day 33 , suggesting that at those times there was minimal risk for donor graft rejection. However, the IR level increased to 1.23 on post-transplant day 75 , corresponding to the period of hyperammonemia. On post-transplant day 102 , the IR measurement was still elevated at 1.19. Donor-specific antibodies were not detected. Immunosuppression was withdrawn and liver transplantation was performed at 11 
months of age. Histologic examination of the explanted liver showed no evidence of radiationinduced damage. Because the patient had been off immunosuppression for some time, we $\underline{\text { thought that the transplanted cells were no longer present by this time and therefore did not }}$ attempt to detect transplanted cells in the explant.

\section{Treatment of a patient with classical PKU}

We next transplanted hepatocytes into a 27 year-old woman with classical PKU owing to two previously characterized null mutations in the phenylalanine hydroxylase gene $(P A H)$. She was noncompliant with her diet, reported severe fatigue, worsening anxiety, and problems with depression. Her mean peripheral blood PHE level was $1343 \pm 48 \mu \mathrm{M}$, in the range for untreated classical PKU. Her full scale IQ was 89. She was Kuvan non-responsive and declined to participate in a trial of enzyme substitution therapy.

Indications for performing hepatocyte transplantation for PKU and the associated risks were discussed extensively among the transplant team, the patient, and her family. After suitable hepatocytes were obtained from the explant of a patient undergoing liver transplant for classical maple syrup urine disease (MSUD), the recipient of the hepatocytes received a single 10Gy fraction of radiation to the right lobe of the liver by gated Intensity Modulated Radiation Therapy; $31 \%$ of the total liver volume was irradiated. This was performed immediately prior to transplant once the quality and quantity of the donor cells was deemed adequate to perform the procedure. Ultimately, only approximately $5.0 \times 10^{9}$ viable hepatocytes $\left(1.0 \times 10^{8}\right.$ viable cells $/ \mathrm{kg}$ body weight; one-half of the target dose) were transplanted over three infusions into the liver. Infusion of this smaller number of donor hepatocytes occurred because the viability of the isolated hepatocytes unexpectedly deteriorated to below the threshold level allowed by protocol during the course of the procedure. Transplantation was performed through the portal vein by 
recanalization of the umbilical vein, which had been used in the two infants that had previously undergone hepatocyte transplantation.

As our studies suggested that hepatocellular graft loss in the first two patients was a result of acute cellular rejection, we elected to measure rejection risk with CD154+ TcM more frequently to assess the effects of altered immunosuppression. In addition, the patient received perioperative anti-lymphocyte antibody therapy. Maintenance immunosuppression consisted of Tacrolimus (maintenance $12-15 \mathrm{ng} / \mathrm{ml}$ for 3 months, then $8-10 \mathrm{ng} / \mathrm{ml}$ ), mycophenolate mofetil (CellCept) as before, and prednisone, which was tapered off over 6-months.

Protocol liver biopsies performed 3 and 6 months post-transplant demonstrated multiple small clusters of transplanted cells by both fluorescence in situ hybridization (FISH) for the $\mathrm{X}$ and $\mathrm{Y}$ chromosomes to identify male donor cells in the female recipient background (Fig. 6A). Immunocytochemistry using an antibody for the PAH enzyme showed clusters of $\mathrm{PAH}+$ cells indicating proliferation of donor hepatocytes (Fig. 6B, C). The biopsy also showed multiple mitoses and Ki67+ cells indicating proliferation of either donor or host hepatocytes (Fig. 6D, E). As post-PCR melting can distinguish donor and host DNA by heteroduplex formation where $A: A$ and T:T mismatch base pairing occurs, this strategy was used to estimate level of engraftment from the liver biopsy. The experimental profile shown in Fig. $6 \mathrm{E}$ indicates the presence of approximately $6 \%$ donor DNA; however, with correction for salt concentration, the actual donor percentage was estimated to be $3 \%$.

Following transplantation, the patient returned to her unrestricted diet and maintained a mean PHE level of $854 \pm 25 \mu \mathrm{M}$ (normal $30-120 \mu \mathrm{M}$; treatment goal $\leq 360 \mu \mathrm{M}$ ) after transplant (Fig. 7A). The drop of PHE by $36 \%$ compared to her pre-transplant levels was statistically significant 
$(p<0.0001)$. Two immune monitoring measurements early after transplantation suggested increased risk of rejection (Fig. 7B). However, this risk appeared to be transient as subsequent measurements were lower. Seven months after transplantation the patient's PHE tolerance abruptly decreased to pre-transplant baseline. As Tacrolimus levels were below our target range, the patient was treated for presumed rejection with corticosteroids and augmented basal immunosuppression with PHE levels subsequently returning to $840 \mu \mathrm{M}$. Interestingly, the IR values for CD154+ TcM indicated increased risk of rejection on three consecutive measurements during this time period, suggesting that the allograft function was impaired due to rejection. A low level of DSA (weak DR53) was identified in the serum on post-operative day 64 and persisted but remained unchanged in intensity when the second peak in IR occurred on post-operative day 183.

PHE levels remained at $840 \mu \mathrm{M}$ for more than a year, but follow-up after this time became inconsistent, and consequently immune monitoring was not performed. As serum PHE levels remained intermittently elevated the functional status of the cellular graft needed to be evaluated. In order to do this, whole body PHE oxidation, an in vivo measure of PAH function, was performed in lieu of a liver biopsy. As measurement of whole body PHE oxidation was not available prior to transplant, the patient's similarly affected brother was studied as a control. The patient and her brother's PHE oxidation were not statistically different (Fig. 8). As a result, immunosuppression was discontinued on the assumption that the graft had been rejected. The patient remains well several months after discontinuation of immunosuppression and she did not experience any side effects of medication withdrawal. 


\section{Discussion}

Over the past twenty years, isolated hepatocytes derived from donor livers not used for organ transplant have been shown to engraft in the liver, to partially correct liver-based inborn errors of metabolism, and extend survival in patients with acute liver failure [1]. However, the degree of replaced function and the period of time clinical improvement has been sustained has not been adequate to circumvent the need for whole organ replacement in patients with liver-based metabolic disorders. Limitations to successful hepatocyte transplantation include difficulties in providing a cell mass large enough to provide clinically significant correction of hepatic function, limited availability of donor organs and hepatocytes, and an inability to monitor the graft in real time for rejection or function. Here we provide the first report using preparative liver-directed radiation therapy, and continuous monitoring for possible rejection in an attempt to overcome these limitations. We also demonstrated in a clinical setting the use of hepatocytes derived from explanted livers following organ transplant, and the importance of carefully measuring replaced hepatic function. In addition, we examined the application of hepatocyte transplantation to treat PKU, a disease that is not life threatening, but that is sub-optimally treated in some adults [2933].

Pre-transplant radiation conditioning of the host liver was added to enhance initial engraftment of hepatocytes and to provide an environment conducive for donor hepatocyte proliferation [9, 34]. We have demonstrated that regional liver-directed irradiation enables selective donor hepatocyte repopulation in individual liver segments and that the effect of radiation on donor hepatocyte expansion is dose dependent $[10,11,18]$. These published studies indicated that delivering 10Gy of radiation to the right liver lobe might allow enough host hepatocyte replacement by donor cells to correct liver-based metabolic deficiencies [26]. In pre-clinical studies in NHPs, we now show that preconditioning of the native liver by irradiation not only 
increases the initial engraftment of xenogeneic hepatocytes, it also creates a microenvironment that allows the engrafted cells to expand to occupy up to $15 \%$ of the irradiated right lobes, which should be sufficient to correct many metabolic diseases of the liver, such as Crigler-Najjar syndrome type I and $\operatorname{PKU}[2,25,26]$. In addition, treatment with a conventionally used regimen of immunosuppression allowed efficient engraftment and survival of xenogeneic hepatocytes for a period of weeks to months.

Liver biopsies in our PKU patient showed transplanted hepatocytes present as small colonies, and that multiple mitoses and Ki67+ hepatocytes were present, findings not seen routinely on liver biopsies from animals or patients undergoing hepatocyte transplant without a pretransplant conditioning regimen. These results indicate that the preparative regimen of liverdirected irradiation induced proliferation of hepatocytes of donor and/or host origin. However, the level of donor hepatocyte replacement was lower than anticipated. Since the majority of donor cells were infused within 24 hours of completion of radiation therapy, a much earlier time than reported in studies involving rodents, it is possible that the potential benefit of radiation conditioning was not completely realized in our patients as optimization of the benefits appears to require separating the radiation treatment and cell infusion by 24 hours [35].

It is important to note that hepatocytes for transplantation can be isolated from a wider array of potential donors than can whole organs, allowing the use of organs that would otherwise be discarded. For example, excellent hepatocyte viability has been demonstrated after their isolation from non-heart-beating donors [36]. The hepatocytes used in the transplant of the PKU patient were isolated from the explant of a patient with a different metabolic liver disease undergoing orthotopic liver transplant, as previously described [8]. Explanted livers from patients with MSUD have been used for domino whole organ transplants, and since only a fraction of the 
host liver is replaced by transplanted hepatocytes, explanted livers from patients with a range of other metabolic liver diseases should be useful as donors for hepatocyte transplantation [37]. There is further potential for ex-vivo manipulation of cells to repair any insults that occur during isolation and storage. In this way hepatocyte transplantation offers potential utility for currently underutilized donor and explanted organs. In this report, we functionally characterized the isolated hepatocytes used for transplantation. However, we have been unable to identify a measure that can predict engraftment, or help decide whether to proceed with hepatocyte infusion with more precision than hepatocyte viability and plating efficiency [38].

In pre-clinical studies, porcine hepatocytes were transplanted into the livers of cynomolgus monkeys. This allowed easy identification of donor hepatocytes within the host liver. In addition, we wished to examine the potential utility of transplanting hepatocyte xenografts in patients. We postulated that hepatocyte xenografts might address the life-threatening manifestations of acute liver failure $[39,40]$, allowing time for regeneration of the native liver to begin. For such an application, permanent engraftment of hepatocytes and replacement of all liver functions is not needed, or necessarily desired. Instead, hepatocyte transplantation need only provide or amplify the several functions that will avert death, such as regulating intracranial pressure, for a limited period of time. Hepatocyte xenografts could be performed as planned procedures, using wellcharacterized sources that are uniform (inbred or even cloned) and potentially engineered genetically to enhance key functions or address important hurdles. Although biological incompatibility and heightened immunity pose barriers [41], those barriers might potentially be overcome by repeated transplants, an approach rarely if ever possible for hepatocyte allografts, and by improvements in immunosuppression that make immunity to xenografts more tractable than previously thought [41-43]. Unlike organ transplants, hepatocyte xenografts are not susceptible to antibody-mediated rejection $[22,44]$ and currently used immunosuppressive 
agents can usually control cell-mediated rejection of xenografts, at least for weeks to months $[22,45]$.

The physiological benefit of hepatocyte xenografts, however, remains to be tested. Because the endogenous liver of the recipients of porcine hepatocyte xenografts in this study had normal function, it has been very difficult to accurately measure most functions of the transplanted cells. However, the hepatocyte xenografts did produce albumin since the porcine protein was detectable in the blood, despite the abundance of the monkey protein. Moreover, when the experiments were terminated, the porcine xenografts expressed appreciable albumin protein for detection and occupied a substantial fraction of liver tissue. The fact that xenogeneic albumin levels did not correspond to levels of engraftment is not surprising as this has been observed even in mouse models of xenogeneic hepatocyte repopulation. Nearly complete repopulation $(>90 \%)$ of FRG or UPA SCID mice with xenogeneic human hepatocytes results in human albumin levels of $<10 \mathrm{mg} / \mathrm{mL}$, levels that are only a fraction of normal mouse albumin levels [46, 47]. The results reported here together with our prior report of hepatocyte xenograft survival exceeding 80 days for individual transplants and 250 days for repeated transplants [22] suggest that current approaches to immunosuppression can allow hepatocyte xenografts to survive long enough to potentially confer a clinical benefit.

An important obstacle to hepatocyte transplantation has been the limited duration of effectiveness, with hepatocytes presumably being lost to immunological rejection [48, 49]. Retrospective analysis of immune reactivity in the two infants with urea cycle defects who underwent hepatocyte transplant, indicates that loss of graft function likely was secondary to rejection, and that failure to provide adequate immunosuppression during the initial donor hepatocyte infusion may have led to graft loss. This finding led us to alter our 
immunosuppression regimen to include induction with an anti-lymphocyte antibody and perform serial immune monitoring in our third patient. Clinical follow up of this patient demonstrated improved PHE levels and evidence of engraftment as long as anti-donor activity, measured by recipient cytotoxic memory $\mathrm{T}$ cells, demonstrated low risk of rejection. Our experience with this assay appears encouraging for following transplanted cells as compared to biopsy, which carries more risk and where it may not be possible to distinguish host from donor cells. The assay provided support for the clinical suspicion of cellular allograft rejection, and correlated nicely with the response to therapy. This is the first report suggesting the benefits of the CD154 assay in cell transplantation, and its use could potentially be a solution to a major obstacle in the field. The utility of DSA quantification in these patients remains unclear, although the appearance of a class 2 antibody was identified in the third patient at a time that corresponded with a peak in the IR. Monitoring for rejection in these patients is hampered by the lack of a known mechanism for rejection in hepatocyte transplant, though normal human hepatocytes are thought to express little class 2 HLA antigens on their surface [50].

Evaluation of donor cell function has been hampered by inadequate methods to quantitate the enzyme activity of transplanted hepatocytes in vivo. Our data show the extent to which PHE measurements were misleading as an indicator of graft viability. Monitoring of PHE levels is considered the mainstay of dietary management in PKU, but levels depend not only on PHE intake, but are also affected by activity, illness, physiologic stress, and total protein balance. Although liver biopsy post-transplant showed clear evidence of engraftment and function on biopsy, consistent with apparent improvement in metabolic control, at a later time point the patient's whole body PHE oxidation was not significantly higher than her untransplanted, affected brother. A similar phenomenon was seen following hepatocyte transplantation for Crigler-Najjar syndrome type I, where daily measures of bilirubin varied more than $30 \%$, making 
it difficult to determine even in that setting whether graft rejection was taking place [2]. These findings emphasize the importance of real-time assays of hepatocyte-specific enzyme activity to assess graft viability, as diagnosing rejection is difficult.

Hepatocyte transplantation for the treatment of PKU has been reported once previously in a 6year-old boy with severe Kuvan nonresponsive PKU not compliant with diet [8]. Following two hepatocyte infusions, blood phenylalanine (PHE) levels fell from a mean of $600 \mu \mathrm{M}$ to a mean of $300 \mu \mathrm{M}$ while the PHE half-life decreased from 43 to 19 hours. In that case, the graft also appeared to be lost from rejection three months after transplant, emphasizing the difficulty in achieving long-term function by transplanted hepatocytes.

Treatment of PKU from birth in infants identified through newborn screening prevents the devastating neurologic problems including seizures and mental retardation [51, 52]. However, patients face life long medical challenges [53]. Neuropsychometric testing has revealed persistent defects in executive function, psychiatric symptoms, and ADHD in these patients [2933, 54]. Adults with poor PHE control can exhibit de novo white matter degeneration, gait disturbances, and seizures [55]. Additionally, early relaxation of diet in adolescence leads to the risk of regression of cognitive function. As a result, alternative therapies are needed.

In experimental studies, increased blood PHE levels do not develop in normal mice until 95\% of their liver has been repopulated with PAH-deficient hepatocytes [26], and PHE levels are completely corrected in mice with PKU if more than $10 \%$ of the liver is replaced with wild-type hepatocytes. Repopulation with fewer than 10\% PAH-positive hepatocytes significantly lowers serum PHE in mice with PKU consuming normal mouse chow. Thus, replacement of about $5 \%$ 
of hepatocytes with normal PAH activity should substantially reduce serum PHE levels in patients with classical PKU. However, benefits of hepatocyte transplant must be weighed against the need for immunosuppressive medication and the associated risks. Fortunately, this risk is low with modern immunosuppressive agents and regimens. Whereas immunosuppression has historically been considered appropriate only in the treatment of life threatening conditions such as whole organ transplants and cancer, current immunosuppressive regimens are safer and applied to conditions such as inflammatory bowel disease, rheumatoid arthritis and psoriasis that impair the quality of, but are not of immediate risk to, life. We believe that PKU has a far more profound impact on the quality of life than most of the conditions for which immunosuppressive medications are now prescribed. Even a modest improvement in metabolic support would make controlling PHE levels with diet more effective, improve neurologic function, and dramatically change the quality of life of both children and adults. The ability to eat a normal or nearly normal diet would be transformational for many patients with PKU. Thus we believe that it is appropriate to consider hepatocyte transplantation for treatment of PKU, even though it is not life threatening. Of course, patient evaluation by the transplant and metabolic treatment team and discussions with the family should be extensive before the procedure is offered. While results from our patient are promising, optimization of the transplant procedure, and the number of cells transplanted are necessary, as is monitoring for immune rejection.

In summary, we report the first systematic testing of liver-directed radiation therapy as a means of improving hepatocyte engraftment. Our pre-clinical studies in immunosuppressed NHPs suggests that radiation preconditioning of the liver enables transplanted hepatocyte xenografts to achieve a sufficient level of engraftment and to maintain that level of engraftment for a sufficient time to confer a physiological benefit, thus offering a solution to two daunting 
obstacles to application of hepatocyte transplantation - the limited availability of hepatocytes and the barriers to engraftment. Our studies on human patients are the first to use preparative radiation prior to hepatocyte transplantation and to use serial assessment of cellular rejection risk by CD154-based T-cell monitoring. Although not fully optimized, these initial studies indicate that radiation preconditioning of the liver is safe, and results in no pathological changes to the liver, even in patients as young as 4 and 7 months. More importantly, the presence of several $\underline{\mathrm{PAH}}+$ clusters indicate that radiation pretreatment induced donor hepatocyte proliferation posttransplantation, a feature needed to improve the efficacy of hepatocyte transplantation. The data involving the use of immune monitoring on these 3 patients also suggests that serial monitoring for cellular rejection risk may be useful for patient management. Optimization of these procedures may produce better engraftment and long-term survival of transplanted hepatocytes than previously reported. Finally, the promising results in the patient with PKU suggests that hepatocyte transplantation should be considered for selected patients with debilitating, but not life-threatening, metabolic diseases.

\section{References}

[1] Soltys KA, Soto-Gutierrez A, Nagaya M, Baskin KM, Deutsch M, Ito R, et al. Barriers to the successful treatment of liver disease by hepatocyte transplantation. Journal of Hepatology 2010;53:769-774.

[2] Fox IJ, Chowdhury JR, Kaufman SS, Goertzen TC, Chowdhury NR, Warkentin PI, et al. Treatment of the Crigler-Najjar syndrome type I with hepatocyte transplantation. New England Journal of Medicine 1998;338:1422-1426.

[3] Dhawan A, Mitry RR, Hughes RD, Lehec S, Terry C, Bansal S, et al. Hepatocyte transplantation for inherited factor VII deficiency. Transplantation 2004;78:1812-1814. 
[4] Meyburg J, Das AM, Hoerster F, Lindner M, Kriegbaum H, Engelmann G, et al. One liver for four children: first clinical series of liver cell transplantation for severe neonatal urea cycle defects. Transplantation 2009;87:636-641.

[5] Stephenne X, Najimi M, Sibille C, Nassogne MC, Smets F, Sokal EM. Sustained engraftment and tissue enzyme activity after liver cell transplantation for argininosuccinate lyase deficiency. Gastroenterology 2006;130:1317-1323.

[6] Sokal EM, Smets F, Bourgois A, Van Maldergem L, Buts JP, Reding R, et al. Hepatocyte transplantation in a 4-year-old girl with peroxisomal biogenesis disease: technique, safety, and metabolic follow-up. Transplantation 2003;76:735-738.

[7] Muraca M, Gerunda G, Neri D, Vilei MT, Granato A, Feltracco P, et al. Hepatocyte transplantation as a treatment for glycogen storage disease type 1a. Lancet 2002;359:317-318.

[8] Stephenne X, Debray FG, Smets F, Jazouli N, Sana G, Tondreau T, et al. Hepatocyte transplantation using the domino concept in a child with tetrabiopterin nonresponsive phenylketonuria. Cell transplantation 2012;21:2765-2770.

[9] Guha C, Sharma A, Gupta S, Alfieri A, Gorla GR, Gagandeep S, et al. Amelioration of radiation-induced liver damage in partially hepatectomized rats by hepatocyte transplantation. Cancer Research 1999;59:5871-5874.

[10] Zhou H, Dong X, Kabarriti R, Chen Y, Avsar Y, Wang X, et al. Single liver lobe repopulation with wildtype hepatocytes using regional hepatic irradiation cures jaundice in Gunn rats. PloS one 2012;7:e46775.

[11] Yamanouchi K, Zhou H, Roy-Chowdhury N, Macaluso F, Liu L, Yamamoto T, et al. Hepatic irradiation augments engraftment of donor cells following hepatocyte transplantation. Hepatology 2009;49:258-267. 
[12] Ashokkumar C, Bentlejewski C, Sun Q, Higgs BW, Snyder S, Mazariegos GV, et al. Allospecific CD154+ B cells associate with intestine allograft rejection in children. Transplantation 2010;90:1226-1231.

[13] Ashokkumar C, Shapiro R, Tan H, Ningappa M, Elinoff B, Fedorek S, et al. Allospecific CD154+ T-cytotoxic memory cells identify recipients experiencing acute cellular rejection after renal transplantation. Transplantation 2011;92:433-438.

[14] Ashokkumar C, Talukdar A, Sun Q, Higgs BW, Janosky J, Wilson P, et al. Allospecific CD154+ T cells associate with rejection risk after pediatric liver transplantation. American Journal of Transplantation 2009;9:179-191.

[15] Scharp DW, Lacy PE, Santiago JV, McCullough CS, Weide LG, Boyle PJ, et al. Results of our first nine intraportal islet allografts in type 1, insulin-dependent diabetic patients. Transplantation 1991;51:76-85.

[16] Dagher I, Nguyen TH, Groyer-Picard MT, Lainas P, Mainot S, Guettier C, et al. Efficient hepatocyte engraftment and long-term transgene expression after reversible portal embolization in nonhuman primates. Hepatology 2009;49:950-959.

[17] Yannam GR, Han B, Setoyama K, Yamamoto T, Ito R, Brooks JM, et al. A nonhuman primate model of human radiation-induced venocclusive liver disease and hepatocyte injury. International Journal of Radiation Oncology • Biology • Physics 2014;88:404-411.

[18] Dawson LA, Ten Haken RK. Partial volume tolerance of the liver to radiation. Seminars in Radiation Oncology 2005;15:279-283.

[19] Kovalic JJ, Thomas PR, Beckwith JB, Feusner JH, Norkool PA. Hepatocellular carcinoma as second malignant neoplasms in successfully treated Wilms' tumor patients. A National Wilms' Tumor Study report. Cancer 1991;67:342-344. 
[20] Lawrence TS, Robertson JM, Anscher MS, Jirtle RL, Ensminger WD, Fajardo LF. Hepatic toxicity resulting from cancer treatment. International Journal of Radiation Oncology • Biology • Physics 1995;31:1237-1248.

[21] Order SE, Donaldson SS. Radiation therapy of benign diseases : a clinical guide, 2nd ed. New York: Springer; 2003.

[22] Nagata H, Nishitai R, Shirota C, Zhang JL, Koch CA, Cai J, et al. Prolonged survival of porcine hepatocytes in cynomolgus monkeys. Gastroenterology 2007;132:321-329.

[23] Phelps CJ, Koike C, Vaught TD, Boone J, Wells KD, Chen SH, et al. Production of alpha 1,3-galactosyltransferase-deficient pigs. Science 2003;299:411-414.

[24] Cooper DK, Good AH, Koren E, Oriol R, Malcolm AJ, Ippolito RM, et al. Identification of alpha-galactosyl and other carbohydrate epitopes that are bound by human anti-pig antibodies: relevance to discordant xenografting in man. Transplant Immunology 1993;1:198-205.

[25] Dhawan A, Puppi J, Hughes RD, Mitry RR. Human hepatocyte transplantation: current experience and future challenges. Nature Reviews Gastroenterology and Hepatology 2010;7:288-298.

[26] Hamman K, Clark H, Montini E, Al-Dhalimy M, Grompe M, Finegold M, et al. Low therapeutic threshold for hepatocyte replacement in murine phenylketonuria. Molecular Therapy 2005;12:337-344.

[27] Lin SS, Weidner BC, Byrne GW, Diamond LE, Lawson JH, Hoopes CW, et al. The role of antibodies in acute vascular rejection of pig-to-baboon cardiac transplants. Journal of Clinical Investigation 1998;101:1745-1756.

[28] Gramignoli R, Dorko K, Tahan V, Skvorak KJ, Ellis E, Jorns C, et al. Hypothermic Storage of Human Hepatocytes for Transplantation. Cell transplantation 2014;23:1143-1151. 
[29] Azen CG, Koch R, Friedman EG, Berlow S, Coldwell J, Krause W, et al. Intellectual development in 12-year-old children treated for phenylketonuria. American Journal of Diseases of Children 1991;145:35-39.

[30] Channon S, Mockler C, Lee P. Executive functioning and speed of processing in phenylketonuria. Neuropsychology 2005;19:679-686.

[31] Greeves LG, Patterson CC, Carson DJ, Thom R, Wolfenden MC, Zschocke J, et al. Effect of genotype on changes in intelligence quotient after dietary relaxation in phenylketonuria and hyperphenylalaninaemia. Archives of Disease in Childhood 2000;82:216-221.

[32] Koch R, Azen C, Friedman EG, Williamson ML. Paired comparisons between early treated PKU children and their matched sibling controls on intelligence and school achievement test results at eight years of age. Journal of Inherited Metabolic Disease 1984;7:86-90.

[33] Weglage J, Pietsch M, Denecke J, Sprinz A, Feldmann R, Grenzebach M, et al. Regression of neuropsychological deficits in early-treated phenylketonurics during adolescence. Journal of Inherited Metabolic Disease 1999;22:693-705.

[34] Guha C, Parashar B, Deb NJ, Garg M, Gorla GR, Singh A, et al. Normal hepatocytes correct serum bilirubin after repopulation of Gunn rat liver subjected to irradiation/partial resection. Hepatology 2002;36:354-362.

[35] Kabarriti R, Zhang W, Yaffe H, Liu L, Asp P, Tome WA, et al. Delaying Transplantation by 24 Hours After Preparative Hepatic Irradiation Enhances Engraftment and Proliferation of Transplanted Hepatocytes in Mouse Liver. Int J Radiat Oncol 2014;90:S174-S175.

[36] Hughes RD, Mitry RR, Dhawan A, Lehec SC, Girlanda R, Rela M, et al. Isolation of hepatocytes from livers from non-heart-beating donors for cell transplantation. Liver Transplantation 2006;12:713-717.

[37] Mazariegos GV, Morton DH, Sindhi R, Soltys K, Nayyar N, Bond G, et al. Liver Transplantation for Classical Maple Syrup Urine Disease: Long-Term Follow-Up in 37 
Patients and Comparative United Network for Organ Sharing Experience. J Pediatr-Us 2012;160:116-U177.

[38] Gramignoli R, Tahan V, Dorko K, Venkataramanan R, Fox IJ, Ellis ECS, et al. Rapid and Sensitive Assessment of Human Hepatocyte Functions. Cell transplantation 2014;23:1545-1556.

[39] Bernal W, Wendon J. Acute liver failure. New England Journal of Medicine 2013;369:2525-2534.

[40] O'Grady JG. Acute liver failure. Postgraduate Medical Journal 2005;81:148-154.

[41] Cascalho M, Platt JL. Challenges and potential of xenotransplantation. In: Rich RR, Fleisher TA, Shearer WT, Schroeder HWJ, Frew AJ, Weyand CM, editors. Clinical immunology: principles and practice, 3rd ed. Philadelphia, PA: Mosby/Elsevier; 2008. p. 1215-1222.

[42] Kleine M, Schrem H, Borlak J, Klempnauer J. Clinical versatility of porcine hepatocytes in the light of interspecies differences in cytochrome $\mathrm{P} 450$ regulation and expression. Xenotransplantation 2008;15:208-217.

[43] Schmelzle M, Schulte Esch J, 2nd, Robson SC. Coagulation, platelet activation and thrombosis in xenotransplantation. Current Opinion in Organ Transplantation 2010;15:212218.

[44] Platt JL. New directions for organ transplantation. Nature 1998;392:11-17.

[45] Bottino R, Wijkstrom M, van der Windt DJ, Hara H, Ezzelarab M, Murase N, et al. Pig-tomonkey islet xenotransplantation using multi-transgenic pigs. American Journal of Transplantation 2014;14:2275-2287.

[46] Azuma H, Paulk N, Ranade A, Dorrell C, Al-Dhalimy M, Ellis E, et al. Robust expansion of human hepatocytes in Fah-//Rag2-///I2rg-/- mice. Nature biotechnology 2007;25:903-910. 
[47] Tateno C, Yoshizane Y, Saito N, Kataoka M, Utoh R, Yamasaki C, et al. Near completely humanized liver in mice shows human-type metabolic responses to drugs. American Journal of Pathology 2004;165:901-912.

[48] Allen KJ, Mifsud NA, Williamson R, Bertolino P, Hardikar W. Cell-mediated rejection results in allograft loss after liver cell transplantation. Liver Transplantation 2008;14:688694.

[49] Jorns C, Nowak G, Nemeth A, Zemack H, Mork LM, Johansson H, et al. De Novo DonorSpecific HLA Antibody Formation in Two Patients With Crigler-Najjar Syndrome Type I Following Human Hepatocyte Transplantation With Partial Hepatectomy Preconditioning. American Journal of Transplantation 2016;16:1021-1030.

[50] Senaldi G, Loboyeo A, Mowat AP, Mielivergani G, Vergani D. Class-I and Class-II Major Histocompatibility Complex Antigens on Hepatocytes - Importance of the Method of Detection and Expression in Histologically Normal and Diseased Livers. J Clin Pathol 1991;44:107-114.

[51] Camp KM, Parisi MA, Acosta PB, Berry GT, Bilder DA, Blau N, et al. Phenylketonuria Scientific Review Conference: State of the science and future research needs. Molecular Genetics and Metabolism 2014;112:87-122.

[52] Vockley J, Andersson HC, Antshel KM, Braverman NE, Burton BK, Frazier DM, et al. Phenylalanine hydroxylase deficiency: diagnosis and management guideline. Genet Med 2014;16:188-200.

[53] Singh RH, Rohr F, Frazier D, Cunningham A, Mofidi S, Ogata B, et al. Recommendations for the nutrition management of phenylalanine hydroxylase deficiency. Genet Med 2014;16:121-131. 
[54] Brumm VL, Azen C, Moats RA, Stern AM, Broomand C, Nelson MD, et al. Neuropsychological outcome of subjects participating in the PKU Adult Collaborative Study: A preliminary review. Journal of Inherited Metabolic Disease 2004;27:549-566.

[55] Landvogt C, Mengel E, Bartenstein P, Buchholz HG, Schreckenberger M, Siessmeier T, et al. Reduced cerebral fluoro-L-dopamine uptake in adult patients suffering from phenylketonuria. Journal of Cerebral Blood Flow \& Metabolism 2008;28:824-831. Author names in bold designate shared co-first authorship

\section{Figure Legends}

Figure 1. Treatment planning for radiation therapy of the patient with classical PKU. Dose distributions along Axial (A), Sagittal (B), and (C) Coronal planes. The patient received a 10Gy dose in a single fraction with 7 fields gated IMRT. The portion of the irradiated right lobe is depicted in red. Light blue, green and deep blue colored isodose lines represent 5Gy, 8Gy, and 10Gy respectively. (D) Dose volume histogram for the patient.

\section{Figure 2. Portal vein access for hepatocyte transplantation in the patient with classical}

PKU. (A) Schematic of hepatocyte infusion into the liver through the portal vein using the recanalized umbilical vein. (B) Photograph of catheter placement into the umbilical vein through a small peri-umbilical incision. (C) Contrast injection demonstrating the occlusion catheter going through the umbilical vein and the left portal vein, which is then directed into the right portal vein. (D) Balloon occlusion and contrast directed into right portal vein. (E) Contrast injection showing "pruning" of the portal venous system following hepatocyte infusion.

Figure 3. Engraftment and proliferation of porcine hepatocyte xenografts in NHPs. (A) Immunofluorescence staining for porcine albumin and $\beta$-Actin in the indicated liver tissue 
specimens. (B) Low power image of porcine albumin stained liver tissues showing large clusters of porcine albumin+ cells proximal to the blood vessels in the right lobe of transplant recipient liver. (C) Quantification of porcine albumin positivity in the left and right liver lobes of each animal. (D) Quantification of porcine albumin positivity in the left and right lobes relative to the whole liver. Values are presented as mean \pm SEM.

Figure 4. Assessment of characteristics of isolated human hepatocytes. Measurement of the viability $(A)$, apoptosis $(B)$, and cytochrome P450 activity and ammonia metabolism (C) of hepatocytes right after isolation and during each round of hepatocyte transplantation.

Figure 5. Schematic presentation of pertinent laboratory investigations in infants that underwent hepatocyte transplants for UCD. (A) In the patient with CPS1 deficiency, elevated baseline IR at the time of transplant and increases in ammonia levels associated with minor increases in protein intake suggests poor graft function or graft failure. (B) In the patient with OTC deficiency, the increase in IR to a level above threshold at day 75 corresponding to an episode of severe ammonia level elevation suggests poor graft function or graft failure.

Figure 6. Histologic findings 6 months post-transplantation in the patient with classical PKU. FISH for the $X$ and $Y$ chromosomes $(A)$ and immunocytochemistry for the PAH enzyme $(\mathrm{B}, \mathrm{C})$ confirming presence of small clusters of donor hepatocytes in the recipient liver. Histology showing mitoses (D) and Ki67+ cells (E) demonstrating proliferation of donor or host hepatocytes. $(F)$ High resolution melt profile indicating the presence of $\sim 6 \%$ donor DNA, that when corrected for salt concentration is estimated to be $3 \%$. 
Figure 7. Changes in peripheral PHE levels and IR levels following hepatocyte transplantation in a patient with PKU. (A) Comparison of PHE levels before and after transplantation showing a drop in PHE level to $854 \pm 25 \mu \mathrm{M}$ (normal 30-120 $\mu \mathrm{M}$; treatment goal $\leq 360 \mu \mathrm{M})$ in the PKU patient on an unrestricted diet after transplant. The decrease in PHE level by $36 \%$ compared to pre-transplant levels was statistically significant. Values are presented as mean \pm SEM and significance was calculated using an unpaired one-tailed t test $\left({ }^{* *} p<0.0001\right)$, followed by an F test to compare variances (ns). (B) PHE and IR levels measured at various time points post-transplant. Spikes in IR levels at 18 and 57 days post-transplant suggested increased risk of rejection but appeared to be transient as subsequent measurements were lower. At 219 days post-transplant, the IR level on three consecutive measurements indicated increased risk of rejection that coincided with an abrupt decrease in the patient's PHE tolerance to pre-transplant baseline, suggesting that the allograft was failing due to rejection. Treatment of the patient with steroids improved PHE tolerance.

Figure 8. Evaluation of cellular graft function by whole body PHE oxidation. Serum PHE levels were intermittently elevated in the PKU patient after more than a year posttransplantation. In order to assess the status of donor hepatocytes, and quantitate the function of transplanted hepatocytes, whole body turnover analysis of $1-{ }^{13} \mathrm{C}$-Phe was performed in lieu of a liver biopsy. As measurement of whole body PHE oxidation was not available prior to transplant, the patient's similarly affected brother was studied as a control. PHE oxidation (presented as a \% of the dose of $1-{ }^{13} \mathrm{C}$-Phe oxidized) in the PKU patient $(\mathrm{A})$ and her brother $(\mathrm{B})$ were both low ( 0.5-0.6\%) and not different. (C) PHE oxidation in a normal control (Note the 50fold change in scale). 

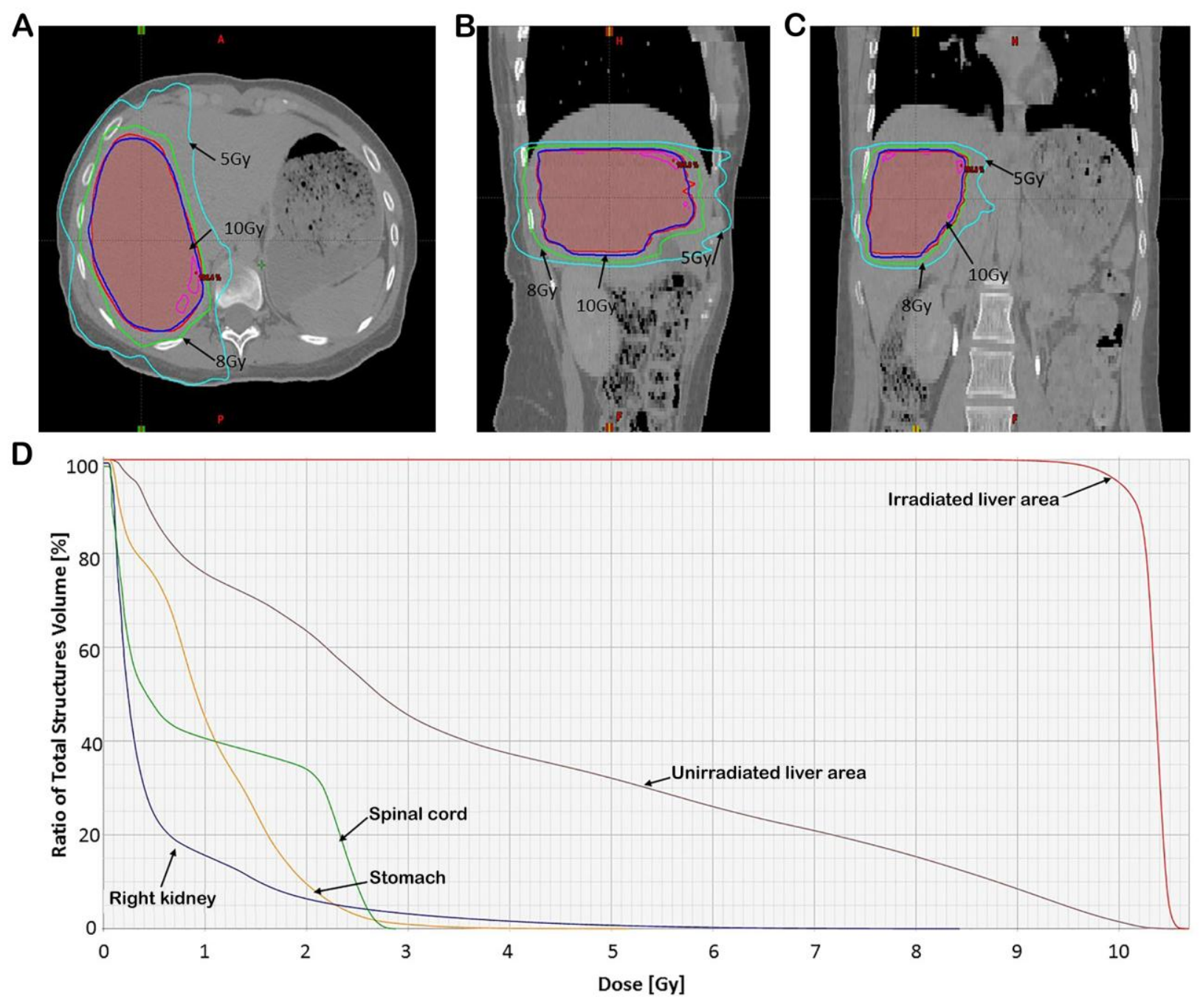
A
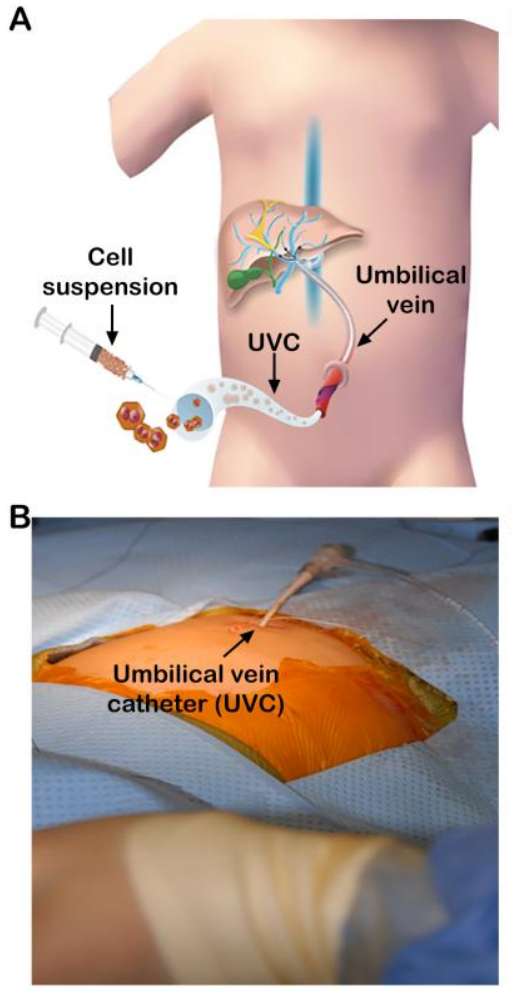

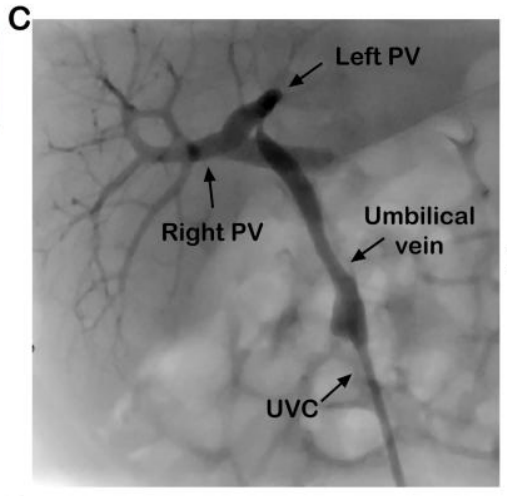

D

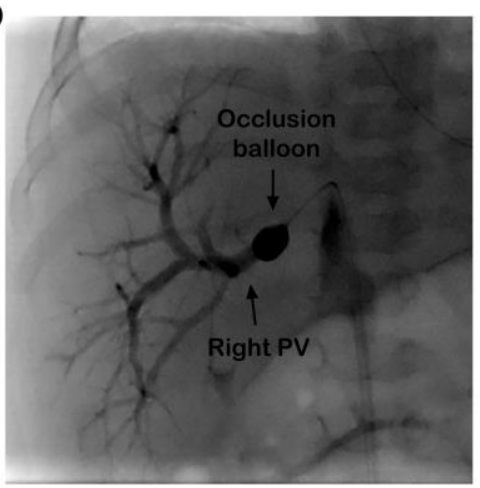

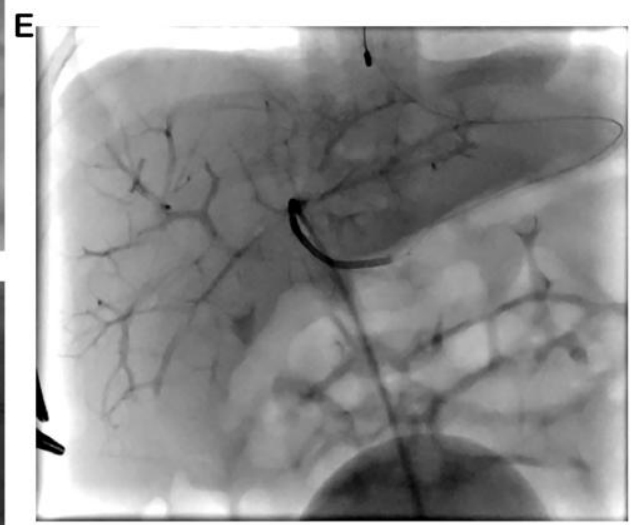



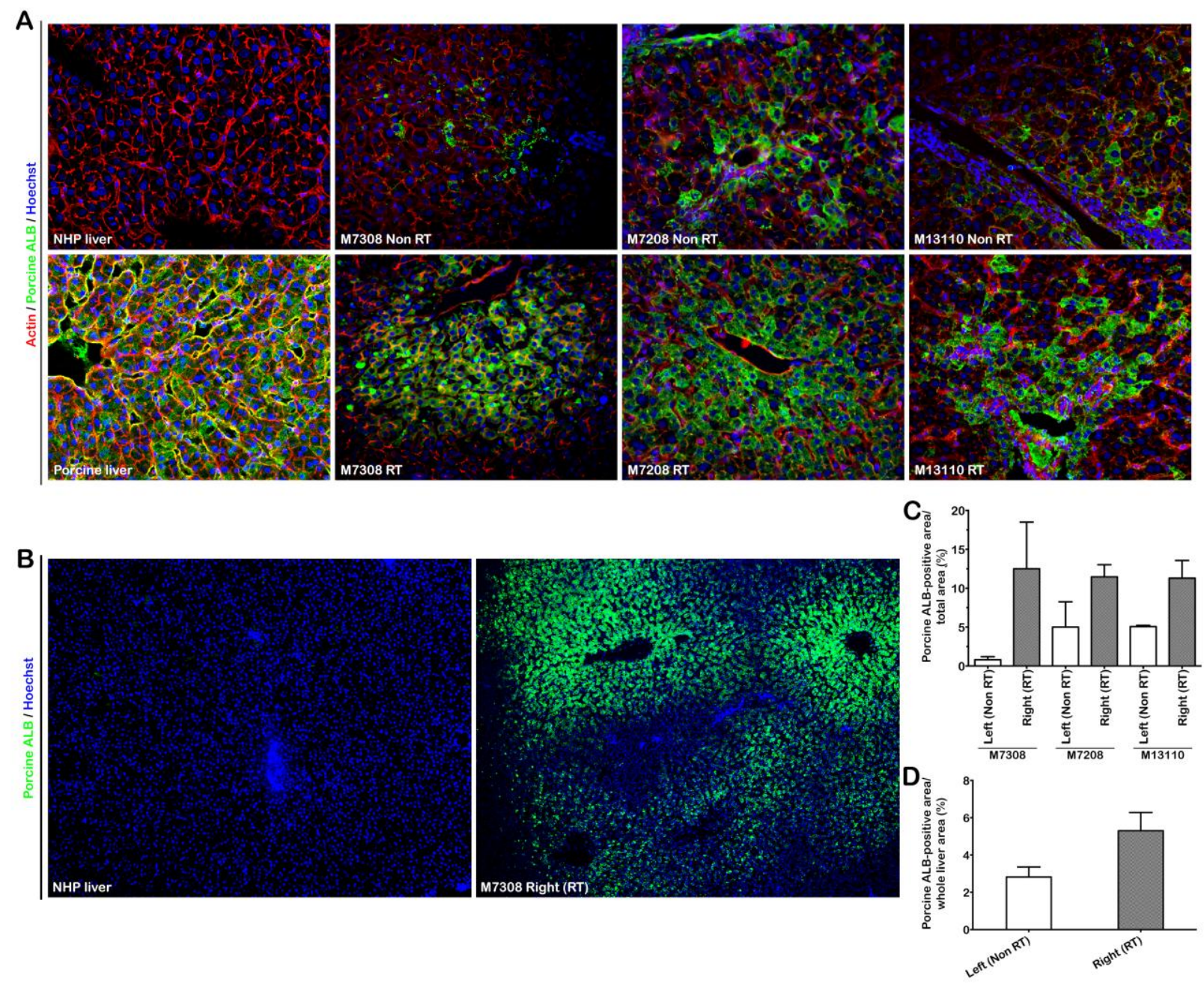
A

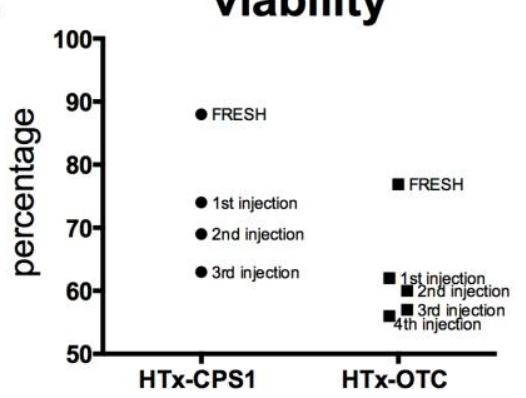

B

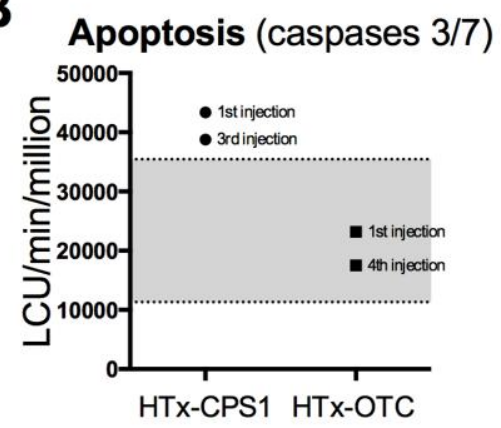

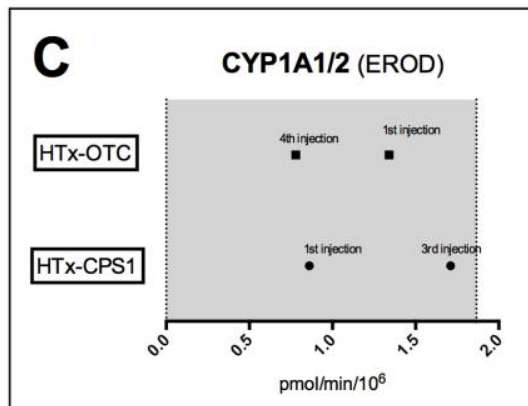
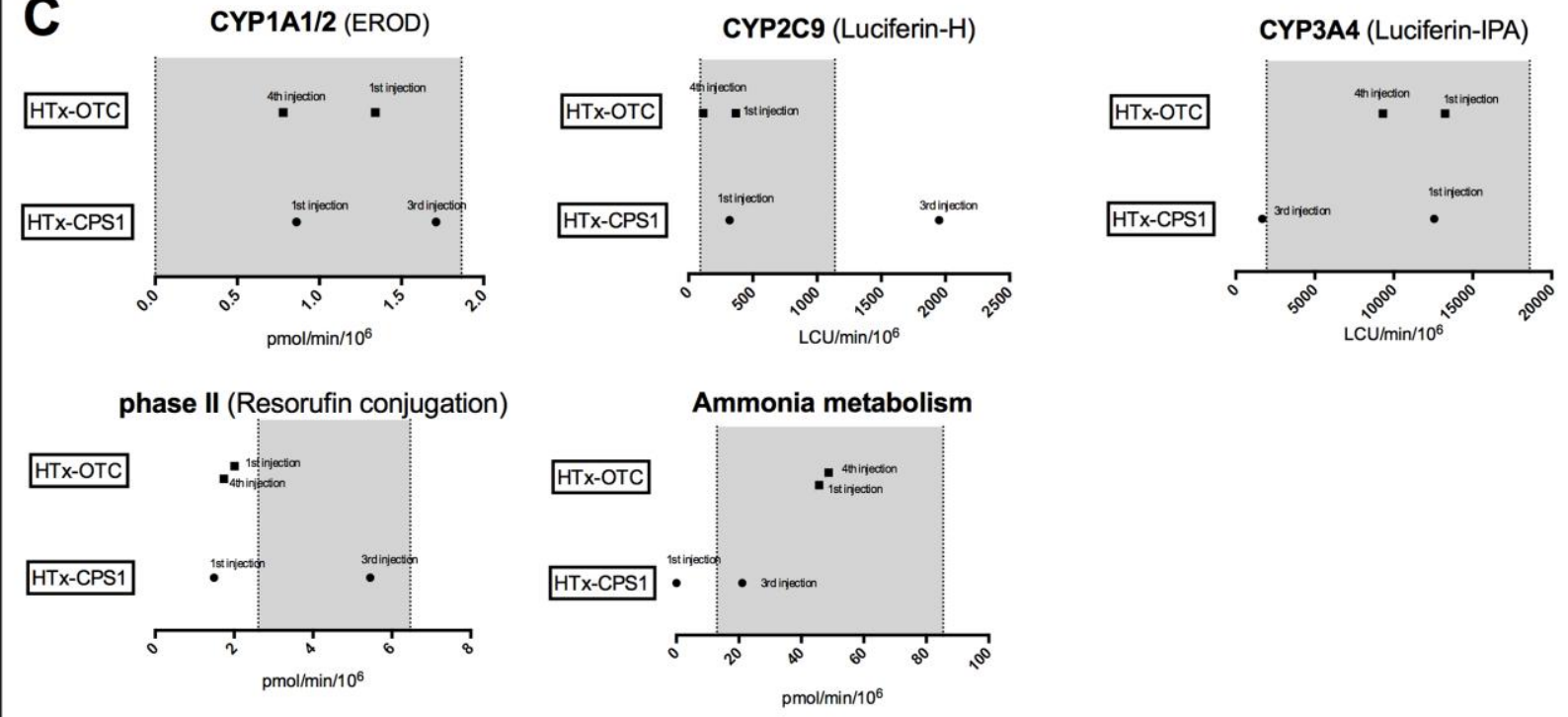

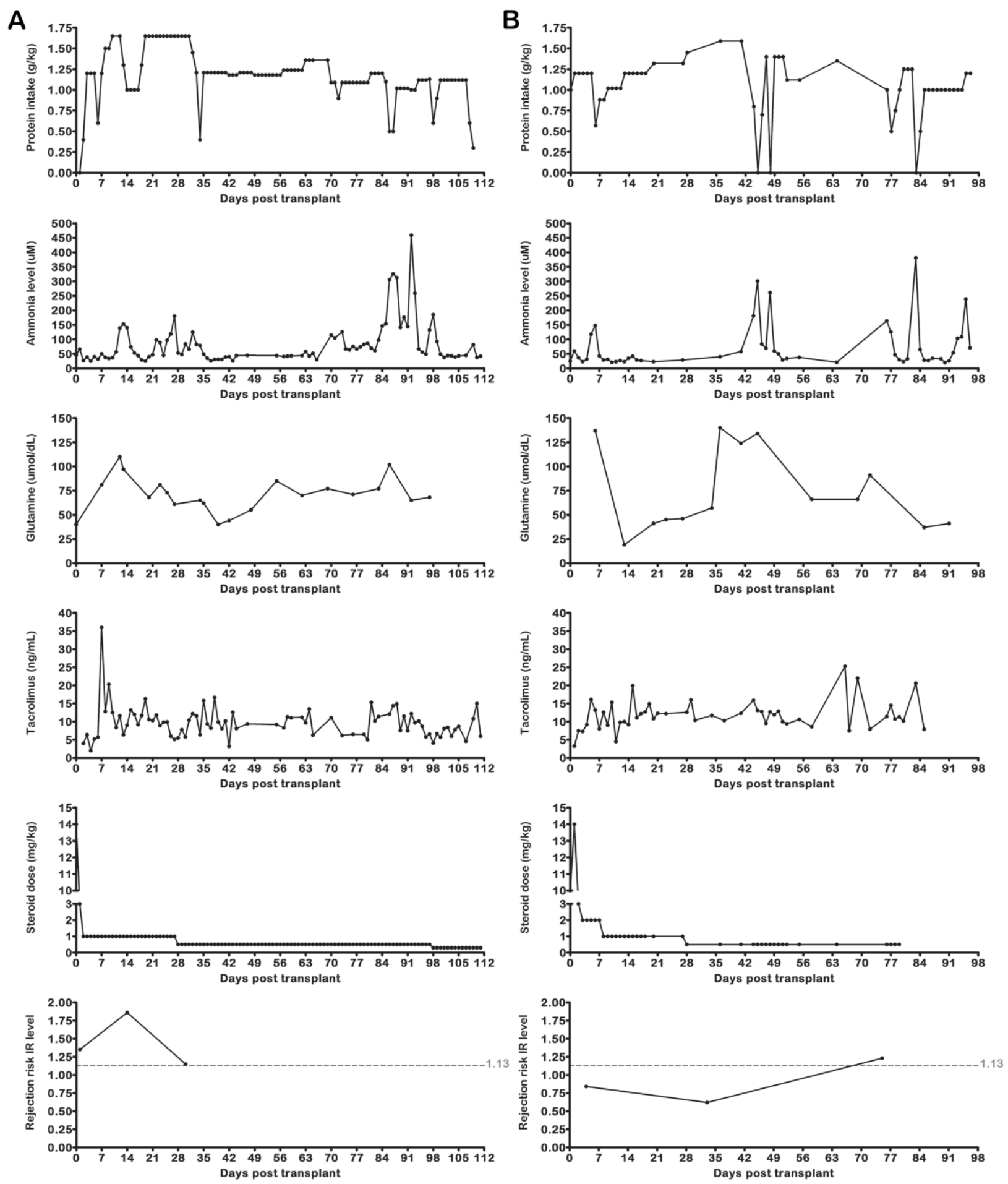

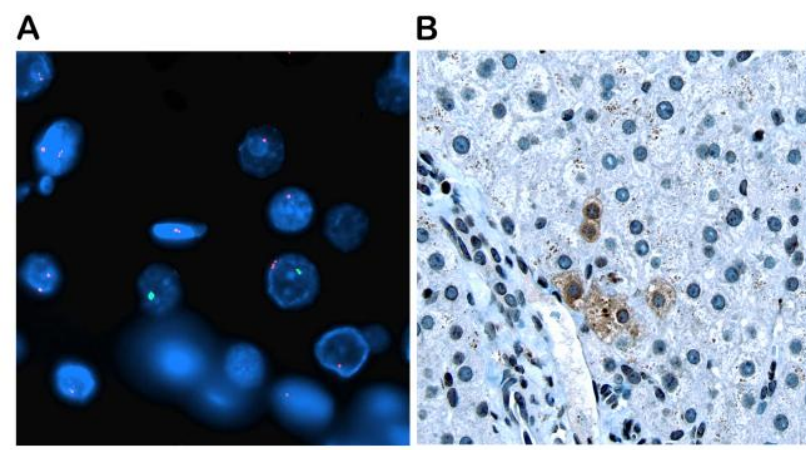

\section{C}

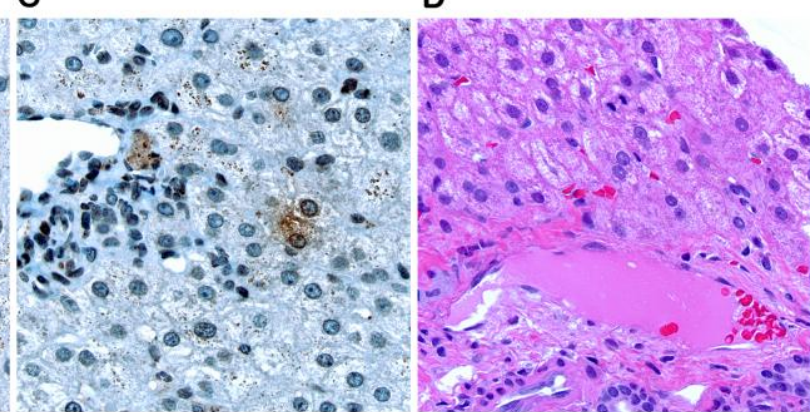

\section{E}

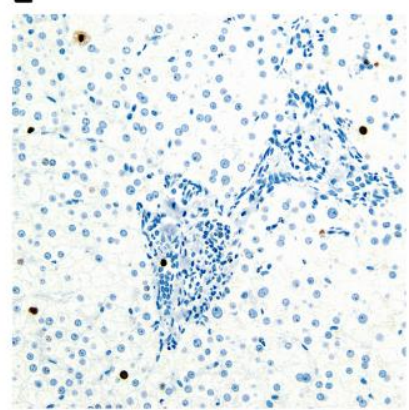

F

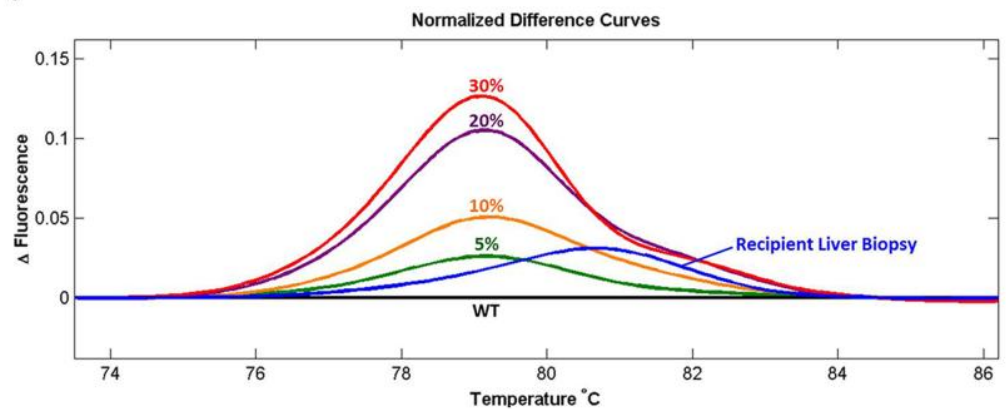




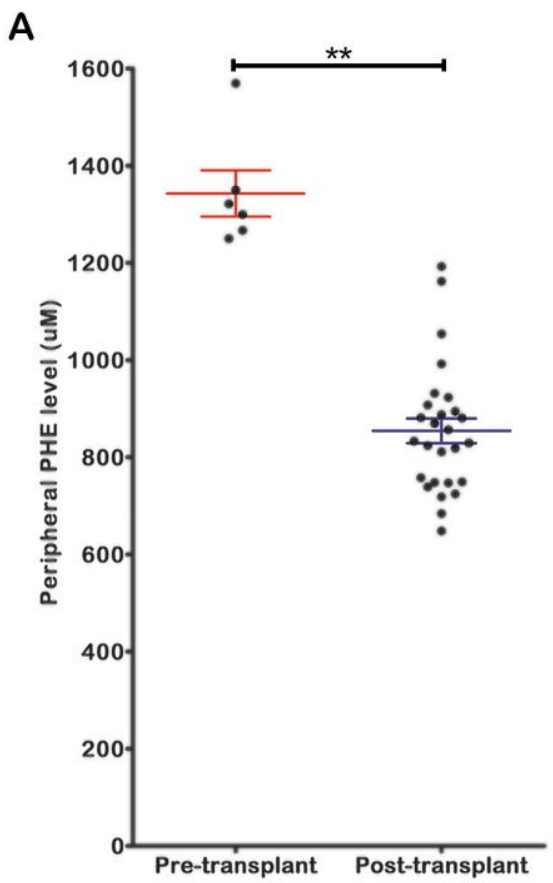

B

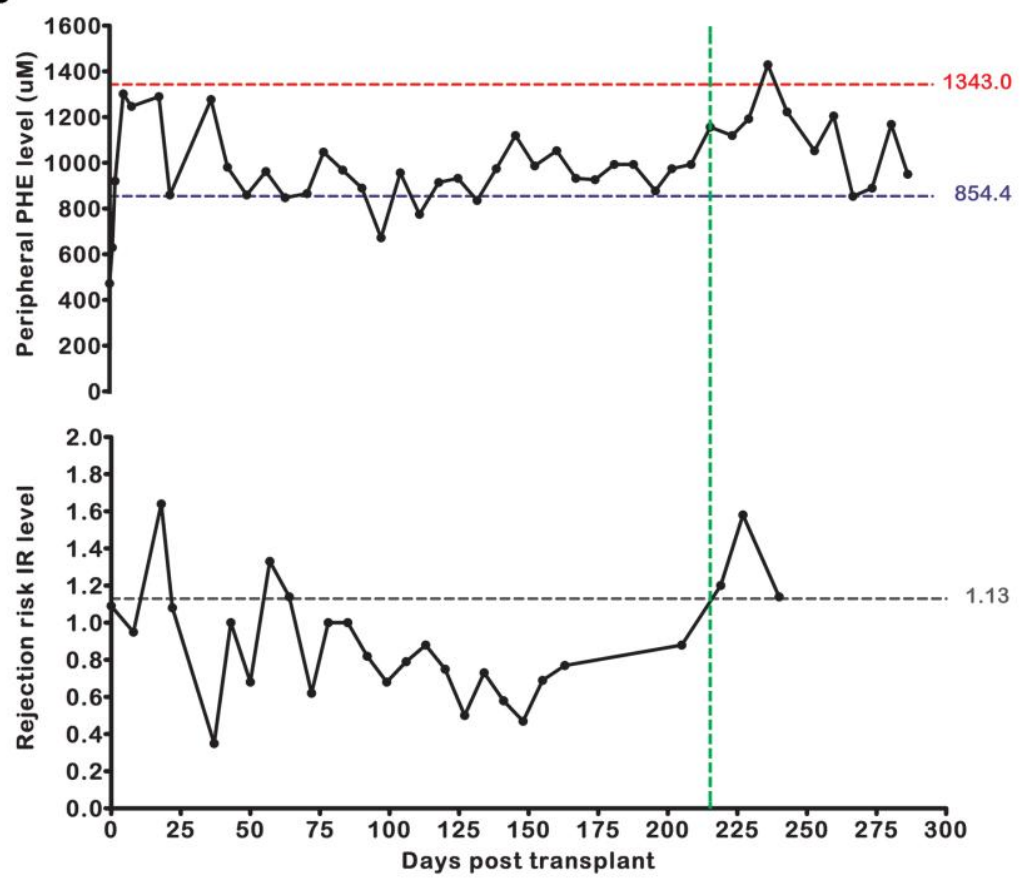



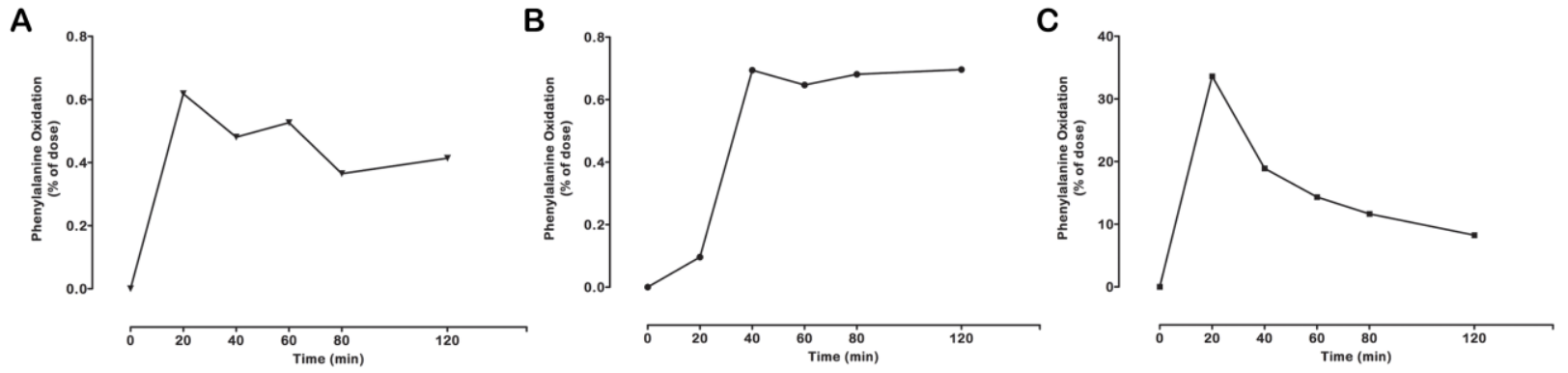


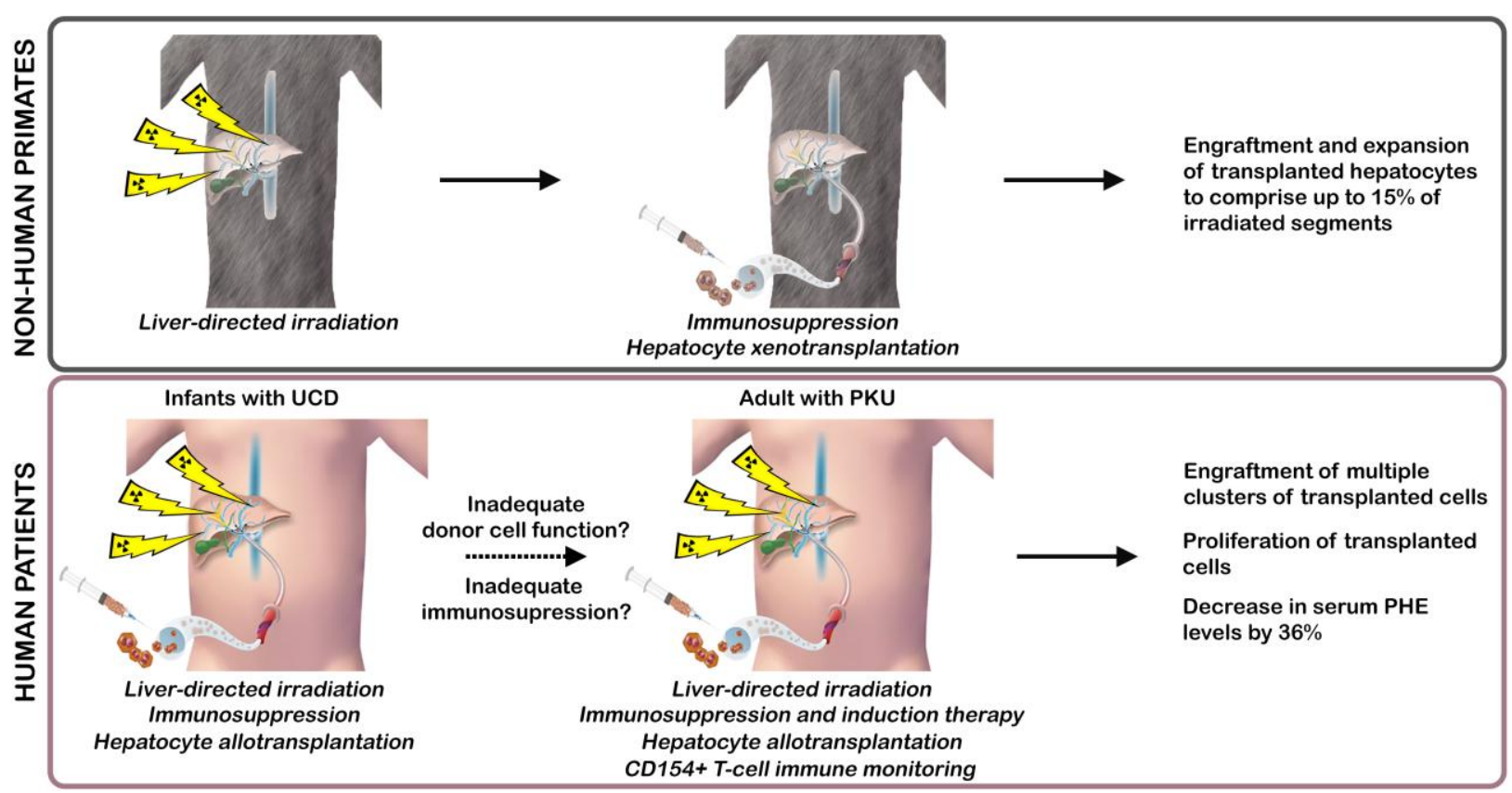

